\title{
Clinical Case of Artesunate Resistant Plasmodium falciparum Malaria in Kolkata: A First Report
}

Bhattacharyya $\mathbf{N}^{1}$, Mukherjee $\mathrm{H}^{2}$, Bose $\mathrm{D}^{3}$, Roy $\mathrm{S}^{4}$, Das $\mathrm{S}^{4}$, Tripathy $\mathrm{S}^{4}$ and Hati $\mathrm{AK}^{5 \star}$

${ }^{1}$ Division of Virology and Medical Entomology, Calcutta School of Tropical Medicine, Kolkata, India

${ }^{2}$ Medical Entomology, Calcutta School of Tropical Medicine, Kolkata, India

${ }^{3}$ Department of Pathology Calcutta Medical College, Kolkata, India

${ }^{4}$ Immunology and Microbiology Laboratory, Department of Human Physiology with Community Health, Vidyasagar University, Midnapore, India

${ }^{5}$ Department of Medical Entomology, Division of Parasitology, Calcutta School of Tropical Medicine, Kolkata, India

\begin{abstract}
The World Health Organization has urged to set up artemisinin-based combination therapy (artemisinin and effective antimalarial drug) and gradually pull out oral artemisinin mono-therapies from the making of prescription due to their high recrudescence rates and to reduce the risk of drug resistance. Prescription practices by physicians and the availability of oral artemisinin monotherapies with pharmacists directly affect the pattern of their use as a result the first clinical case report of only artesunate resistant $P$. falciparum malaria has been found from Kolkata. A significant failure rate of artesunate and SP $(9.5 \%)$ has recently been observed in the district Jalpaiguri, West Bengal, though no specific mutation has been observed in pfATPase6 gene. In view of the above findings to get a clear picture of artemisinin resistance in $P$. falciparum a thorough study is required involving in vivo, in vitro and molecular procedures in this vulnerable area.
\end{abstract}

Keywords: Malaria; Plasmodium falciparum; Artisunate; ACT; Drug resistant

\section{Introduction}

The World Health Organization (WHO) recommends the use of artemisinin derivatives only in combination with efficient partner drugs against Plasmodium falciparum malaria to make sure high cure rates and postponement resistance. Artemisinins produce rapid therapeutic response, reduce gametocyte carriage, and are well tolerated by patients [1]. The five formulations of Artimisinin Combination Therapy (ACT) are artemether+lumefantrine, artesunate+mefloquine, artesunate+amodiaquine, artesunate+sulphadoxine-pyrimethamine and dihydroartemisinin+piperaquine [2]. The ACT was introduced by the National Vector Borne Disease Control Programme (NVBDCP) as the first-line treatment of falciparum malaria in 117 districts which represented more than $90 \%$ of the reported P. falciparum cases [3]. Presently all formulations of ACT recommended by WHO, except dihydroartemisinin+piperaquine, are registered with the Drug Controller General of India to treat the patient as well as to overcome the resistant property of $P$. falciprum.

Now, the success of a new treatment policy is dependent on the adherence of health providers to the guideline and the compliance of patients with the recommended treatment. Equally, information on current provider and patient practices is needed to inform and improve future treatment policy [4]. But our recent report suggests the inappropriate treatment practices continue in spite of the availability of evidenced-based guidelines.

\section{Case Report}

This report stated an in vivo artesunate resistant Plasmodium falciparuum case in Kolkata, capital of West Bengal (Figure 1). A female patient (SL), 57 years of age, was a resident of central Kolkata, West Bengal, India. The patient was suffering from fever with chill and rigor on alternate days from $20^{\text {th }}$ October, 2012. She examined her blood on 26.10.2012 following the advice of her local physician for malaria parasite and dual antigen (PF/PV HRP2/LDH) tests in the Gautam Laboratories, 9A,K K Tagore street, Kolkata 700007. Both thick (Giemsa stained) and thin (Leishman stained) films of the patient were observed. Malaria antigens were tested applying malaria antigen

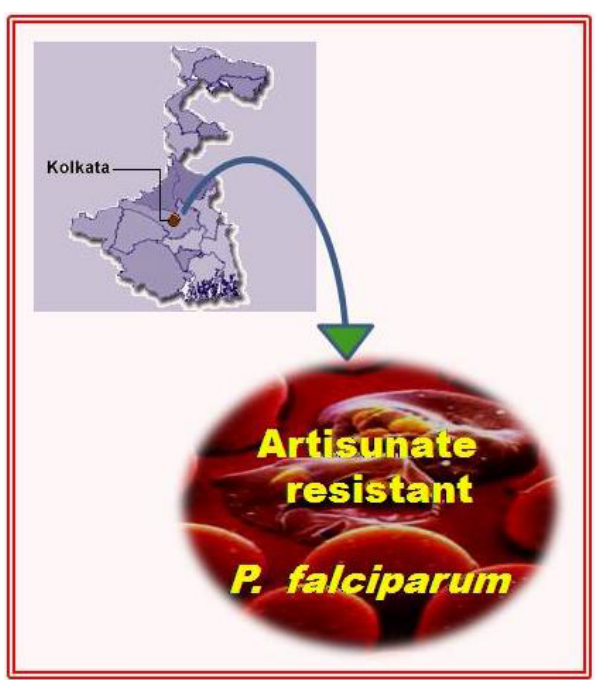

Figure 1: Area of Kolkata in the map of West Bengal.

*Corresponding author: Amiya Kumar Hati, Department of Medical Entomology Division of Parasitology, Calcutta School of Tropical Medicine, Kolkata, India, Tel: 03325773409; Fax: 9103222-275329; E-mail: akhati2012@gmail.com

Received December 16, 2013; Accepted January 04, 2014; Published January 07, 2014

Citation: Bhattacharyya N, Mukherjee H, Bose D, Roy S, Das S, et al. (2014) Clinical Case of Artesunate Resistant Plasmodium falciparum Malaria in Kolkata: A First Report. J Trop Dis 2: 128. doi: 10.4172/2329-891X.1000128

Copyright: (C) 2014 Bhattacharyya N, et al. This is an open-access article distributed under the terms of the Creative Commons Attribution License, which permits unrestricted use, distribution, and reproduction in any medium, provided the original author and source are credited. 
rapid diagnostic test kit of MicroGene Diagnostic systems (P) Ltd., (web: www.microgenediagnostic.com, following the instructions of the company).

Microscopic observation suggested the presence of $P$. falciparum rings in her blood and P. falciparum antigen was also detected. Then she was treated by her physician with artesunate (Falcigo) injection alone, $120 \mathrm{mg}$ i.m. on the first day (26.10.12) followed by $60 \mathrm{mg}$ i.m. daily for the next four days. She became a febrile on and from 28.10.2012. Her blood was tested on 6.11.2012, when no malaria parasite was found.

But, on $19^{\text {th }}$ November, 2012, the patient was attacked by fever. Again her blood was examined on 20.11.12. P. falciparum rings were present and falciparum antigen was also found to be positive. In this time the membrane surface protein test suggests that the patient was recurrence of infection and not the re-infection. This time she was treated with six tablets of artemether $80 \mathrm{mg}$ and lumefantrine $480 \mathrm{mg}$ (Lumether forte DT) from 20.11.2012 administered orally at $0,6,24$, 36,48 and $60 \mathrm{hr}$. She became a febrile from 21.11.2012 and her blood was examined on 21.11.2012, 22.11.2012, 23.11.2012, 29.11.2012, 13.12.2012 and 25.12.2012 and in all the occasions malaria parasite was not found in her blood. She had no history of movement to neighboring countries in recent years.

These results demonstrated that the patient was clinically resistant to artesunate. It was a late treatment failure case. However, the combined therapy (artemether and lumefantrine) was effective in her case, as though the exact protocol was not maintained, in her blood no malaria parasite was found on day 7, 21 and 36, after completion of her treatment with artemether and lumefantrine combined therapy.

\section{Discussion}

This is the first clinical case report of only artesunate resistant $P$. falciparum malaria from Kolkata. Though the present national drug policy is ACT (artemisinin and SP) [5] that has not been followed in this particular case. It is possible that such practices are continuing even with ACT. A significant failure rate of artesunate and SP (9.5\%) has recently been observed in the district Jalpaiguri, West Bengal, though no specific mutation has been observed in $p f A T P a s e 6$ gene [6]. In view of the above findings to get a clear picture of artemisinin resistance in $P$. falciparum a thorough study is required involving in vivo, in vitro and molecular procedures in this vulnerable area. Rationality of inclusion of $\mathrm{SP}$ in ACT also raises a serious question as among isolates from Kolkata most common mutations in $d h f r$ gene (responsible for pyrimethamine resistance) are at positions 108 and 51. The mutation is also found in C59R [7]. Again, in Kolkata $36.67 \%$ of isolates contains quadruple $d h p s$ AGEAT mutation $(436+437+540+613)$, responsible for sulphadoxine resistance [8]. This case suggests that combination therapy effective as well as artesunate and lumefantrine in such cases is more practicable and efficacious than any other combination.

\section{References}

1. Valecha N, Joshi H, Mallick PK, Sharma SK, Kumar A, et al. (2009) Low efficacy of chloroquine: time to switchover to artemisinin-based combination therapy for falciparum malaria in India. Acta Trop 111: 21-28.

2. World Health Organization (2010) Guidelines for the treatment of malaria, second edition.

3. National Vector Borne Disease Control Programme. Directorate General of Health Services Ministry of Health \& Family Welfare.

4. Wasunna B, Zurovac D, Goodman CA, Snow RW (2008) Why don't health workers prescribe ACT? a qualitative study of factors affecting the prescription of artemether-lumefantrine. Malar J 7:29.

5. Guideline for diagnosis and treatment of malaria in India (2009) Government of India.

6. Saha P, Guha SK, Das S, Mullick S, Ganguly S, et al. (2012) Comparative efficacy of artemisinin combination therapies (ACTs) in P. falciparum malaria and polymorphism of pf.ATpase 6, pfacrt. pfdhfr and pfdhps genes in tea gardens of Jalpaiguri district, India. Antimicrob. agents and Chemother 56: 2511-2517.

7. Das S, Chakraborty SP, Tripathy S, Hati AK, Roy S (2012) Association between prevalence of pyrimethamine resistance and double mutation in pfdhfr gene in West Bengal, India. Asian Pacific J Trop Dis 5: 31-35.

8. Das S, Chakraborty SP, Tripathy S, Hati A, Roy S (2012) Novel quadruple mutations in dihydropteroate synthase genes of Plasmodium falciparum in West Bengal, India. Trop Med Int Health 17: 1329-1334. 